\title{
BMJ Open Reducing unnecessary vitamin testing in general practice: barriers and facilitators according to general practitioners and patients
}

\author{
H. Hofstede (D) , ${ }^{1}$ H.A.M. van der Burg, ${ }^{2}$ B.C. Mulder, ${ }^{3}$ A.M. Bohnen, ${ }^{2}$ \\ P.J.E. Bindels, ${ }^{2}$ N.J. de Wit, ${ }^{1}$ E.I.T. de Schepper, ${ }^{2}$ S.F. van Vugt ${ }^{1}$
}

To cite: Hofstede $\mathrm{H}$, van der Burg H.A.M, Mulder B.C, et al. Reducing unnecessary vitamin testing in general practice: barriers and facilitators according to general practitioners and patients. BMJ Open 2019;9:e029760. doi:10.1136/ bmjopen-2019-029760

- Prepublication history for this paper is available online. To view these files, please visit the journal online (http://dx.doi org/10.1136/bmjopen-2019029760).

Received 10 February 2019 Revised 14 August 2019 Accepted 19 August 2019

Check for updates

(c) Author(s) (or their employer(s)) 2019. Re-use permitted under CC BY-NC. No commercial re-use. See rights and permissions. Published by BMJ.

${ }^{1}$ Department of General Practice, Julius Center for Health Sciences and Primary Care, University Medical Center Utrecht, Utrecht, The Netherlands

${ }^{2}$ Department of General Practice, Erasmus MC, University Medical Center Rotterdam, Rotterdam, The Netherlands

${ }^{3}$ Strategic Communication Group, Wageningen University, Wageningen, The Netherlands

Correspondence to

Dr S.F. van Vugt;

s.f.vanvugt@umcutrecht.nl

\section{ABSTRACT}

Objective There has been an increase in testing of vitamins in patients in general practice, often based on irrational indications or for non-specific symptoms, causing increasing healthcare expenditures and medicalisation of patients. So far, there is little evidence of effective strategies to reduce this overtesting in general practice. Therefore, the aim of this qualitative study was to explore the barriers and facilitators for reducing the number of (unnecessary) vitamin $\mathrm{D}$ and $\mathrm{B}_{12}$ laboratory tests ordered.

Design and setting This qualitative study, based on a grounded theory design, used semistructured interviews among general practitioners (GPs) and patients from two primary care networks (147 GPs, 195000 patients). These networks participated in the Reducing Vitamin Testing in Primary Care Practice (REVERT) study, a clustered randomized trial comparing two de-implementation strategies to reduce test ordering in primary care in the Netherlands.

Participants Twenty-one GPs, with a maximum of $1 \mathrm{GP}$ per practice who took part in the REVERT study, and 22 patients (who were invited by their GP during vitaminrelated consultations) were recruited, from which $20 \mathrm{GPs}$ and 19 patients agreed to participate in this study. Results The most important factor hampering vitamintest reduction programmes is the mismatch between patients and medical professionals regarding the presumed appropriate indications for testing for vitamin $\mathrm{D}$ and $\mathrm{B}_{12}$. In contrast, the most important facilitator for vitamin-test reduction may be updating GPs' knowledge about test indications and their awareness of their own testing behaviour.

Conclusion To achieve a sustainable reduction in vitamin testing, guidelines with clear and uniform recommendations on evidence-based indications for vitamin testing, combined with regular (individual) feedback on test-ordering behaviour, are needed. Moreover, the general public needs access to clear and reliable information on vitamin testing. Further research is required to measure the effect of these strategies on the number of vitamin test requests.

Trial registration number WAG/mb/16/039555.

\section{Strengths and limitations of this study}

- This is the first study using semistructured interviews to explore the barriers and facilitators for reducing the number of (unnecessary) vitamin $D$ and $B_{12}$ laboratory tests ordered.

- A qualitative approach with the use of open coding allows all different aspects behind the complexity of reducing vitamin testing to be addressed.

- Potential bias due to selection of general practitioners (GPS) affiliated to a research network and selection of patients by their GPs.

\section{INTRODUCTION}

The number of vitamin tests ordered in general practice has increased substantially in developed countries in recent years. ${ }^{1}$ For example, the regional number of test requests for vitamin $B_{12}$ in Utrecht, the Netherlands, increased almost sixfold between 2004 and $2014 .{ }^{2}$ Vitamin D was the fifth most common laboratory test ordered for Medicare patients in the USA in 2016, at a total cost of US $\$ 350$ million. ${ }^{3}$

Most indications for these tests are probably not evidence based, as a causal relationship with vitamin deficiencies for most health conditions is not present. ${ }^{45}$ This overtesting could result in overdiagnosis and overtreatment with vitamin supplements, further increasing medicalisation, increasing healthcare costs and irrational health perceptions. ${ }^{15-8}$ For example, previous research concluded that, although vitamin testing may potentially be useful in some high-risk groups, overtesting and overtreatment of vitamin $\mathrm{D}$ by general practitioners (GPs) resulted in professional and societal medicalisation of vitamin $\mathrm{D} .{ }^{9}$ To counter this inappropriate medicalisation, a long-term strategy to reduce overtesting and oversupplementation is needed. ${ }^{910}$ 
So far, there is little evidence of effective strategies to reduce this overtesting in general practice, although clinical decision support rules seem promising. ${ }^{11}$ Understanding barriers to, and facilitators for, reducing overtesting is essential to develop a long-term strategy to tackle this problem. ${ }^{10}$ For instance, Moynihan et al suggested that 'commercial and professional vested interests' and 'cultural beliefs that more is better' are facilitators of diagnostic testing that can lead to overdiagnosis. ${ }^{8}$ Furthermore, a qualitative study examining GPs' hidden motives in diagnostic decision making concluded that patients' reassurance was a strong motivation for GPs to perform or order diagnostic tests. ${ }^{12}$ Next to GP-related factors, many patient-related factors may influence clinical decision. ${ }^{13}$

So far, theoretical perspectives, as well as empirical studies on the barriers and facilitators of vitamin test ordering, in general practice are lacking. Therefore, we performed a qualitative assessment using semistructured interviews among both GPs and patients to explore the barriers and facilitators for reducing the number of unnecessary ordered vitamin $\mathrm{D}$ and $\mathrm{B}_{12}$ laboratory tests.

\section{METHODS \\ Design and setting}

Standards for Reporting Qualitative Research reporting guidelines were used for this qualitative study. ${ }^{14}$ This qualitative study used a grounded theory design, ${ }^{15}$ because this design is explicitly suited for examining how meanings in people's perceptions are related to their actions. Applied to our study, using grounded theory allowed us to study how meanings attached to vitamin testing inter-relate to choices and actions regarding vitamin testing for both GPs and patients. The aim was, ultimately, to develop new theoretical concepts, grounded in qualitative data, which represent barriers and facilitators for vitamin testing. These new theoretical concepts may be further developed and tested in future research.

Data were collected through semistructured interviews among GPs and patients from two primary care networks in the Netherlands that participated in the Reducing Vitamin Testing in Primary Care Practice (REVERT) study. The REVERT study was a randomised controlled trial (RCT) assessing the effectiveness of a GP intervention programme including education, monitoring and feedback on numbers in relation to ordering vitamin $\mathrm{D}$ and $\mathrm{B}_{12}$ tests. Four times a year, GPs received feedback on the number of tests they ordered. After randomisation, half of all participating practices also received patient information on vitamin testing. In total, 22 general practices (117 GPs with 134000 patients) in the Utrecht region and four health centres (41 GPs and 61000 patients) in the Rotterdam region participated in the REVERT study.

This study was deemed by the University Medical Center Utrecht ethics committee not to be subject to full assessment.

\section{Recruitment of participants}

At the end of the 1-year intervention period, we have invited all participating general practices for an interview by telephone or face to face by one of the researchers. To secure an adequate case mix regarding practice type and socioeconomic status (SES) of the practice area, only one GP per REVERT practice was invited for an interview.

Patients were recruited through the participating GPs; GPs were asked to invite patients during consultations in which vitamin testing was a topic of conversation. The GPs asked them if they were willing to be interviewed about vitamin testing. When patients consented to be interviewed on this topic, GPs provided the patients' name and telephone number to the researchers, who contacted the patients. We aimed to recruit a mixed sample in terms of age, gender, ethnicity and educational level, because a large variation as to demographic characteristics helps to recruit a sample with the widest range of possible experiences, opinions and preferences. This is necessary for a full exploration of this issue.

\section{Data collection}

The interviews were performed by two interviewers $(\mathrm{HH}$ and $\mathrm{HvdB}$ ) during the last quartile of the intervention period of the REVERT study. The interviewers were two master's medical students with a background in medical research and/or qualitative research, supported by a multidisciplinary team of researchers, GPs and a psychologist who specialised in communication research (BM). $\mathrm{BM}$ trained $\mathrm{HH}$ and $\mathrm{HvdB}$ on how to apply guidelines for doing in-depth interviews.

The GP interviews were conducted face to face in the GPs' office, and the patient interviews were conducted by telephone. Interviews lasted approximately 30 and $15 \mathrm{~min}$ for GPs and patients, respectively, and were semistructured using a list that covered four broad topics of barriers and facilitators for reducing the number of (unnecessary) vitamin $\mathrm{D}$ and $\mathrm{B}_{12}$ testing. The four topics were based on the framework by Grol et al, ${ }^{16}$ namely, (1) perceptions of, and reasons for, vitamin $\mathrm{D}$ and $\mathrm{B}_{12}$ testing; (2) cognitive, motivational and social factors potentially influencing the number of vitamin tests ordered ${ }^{16}$; (3) evaluation of the study intervention (e-module, education and feedback); and (4) ideas regarding a successful strategy for a durable reduction in vitamin test ordering. Baseline characteristics of GPs (sex, age and years working as GP), intervention group (deimplementation strategy 1 or 2) and patients (sex, age and education level) were ascertained at the end of the interview. Data on the number of patients per practice were retrieved by emailing the practices. In addition, data on SES were retrieved from the Social and Cultural Planning Office (SCP) in the Netherlands and were linked to our data through the four digits of the postal codes of the practice area. SCP calculates SES scores based on information concerning education, income and position in the labour market. ${ }^{17}$ We expected interviews with $20 \mathrm{GPs}$ and 20 patients to be sufficient for item saturation. ${ }^{18}$ During data collection, 
interim meetings were held with the interviewers $(\mathrm{HH}$ and $\mathrm{HvdB}$ ) and psychologist (BM) to discuss data and to monitor progress towards saturation.

Based on a previous study, we expected a minimum of approximately 12 interviews with GPs and 12 interviews with patients to be sufficient for saturation, ${ }^{18}$ although numbers mentioned in the literature vary and thus cannot be taken as absolute indicators of saturation or any other criterion. To guarantee at least 12 interviews per group, the aim was to organise about 20 interviews with GPs and 20 interviews with patients. Twenty-one GPs from different practices were invited to participate. One GP declined, so in total, 20 GPs agreed to participate in this study (5 GPs in Rotterdam and 15 GPs in Utrecht). Of the 22 patients who consented to participate in the study, 3 could not be reached by telephone by the researchers, resulting in 19 interviewed patients.

\section{Data analysis}

The interviews were recorded on audiotape and transcribed verbatim. Next, these data were coded, combining a deductive (ie, Grol et al $l_{\text {s framework) }}{ }^{16}$ and an inductive (ie, data-driven) approach using QSR NVivo V.11. ${ }^{19}$ All interviews were coded independently by two researchers ( $\mathrm{HH}$ and $\mathrm{HvdB})$. The emerging themes were continuously compared with interview transcripts. During data collection, interim meetings were held with the interviewers $(\mathrm{HH}$ and $\mathrm{HvdB}$ ) and the communication researcher (BM) to discuss data collection and analysis, including emerging themes and how these inter-related.
The assigned codes and themes were discussed by the coding researchers until consensus was achieved.

Data saturation was monitored and discussed as well. After coding 14 interviews for the GP group and 14 interviews for the patient group, no new codes were added, which means that data saturation was reached at that point.

\section{Patient and public involvement}

Patients and the public were not involved in the design, recruitment and conduct of the study.

\section{RESULTS}

\section{Participants}

The characteristics of the 20 GPs and 19 patients who participated in the study are summarised in table 1.

\section{GPs' reasons for testing}

Two categories of reasons for testing could be distinguished: (1) medical reasons and (2) non-medical reasons. These reasons for testing were influenced by (3) participation in the REVERT study.

\section{Medical reasons}

Patients considered to be at high-risk of vitamin D deficiency (eg, a dark skin) were most often mentioned as a medical reason. Medical reasons for testing vitamin $B_{12}$ levels were a low haemoglobin level, neuropathic symptoms and a potentially insufficient diet. GPs reported testing vitamin D levels for non-specific symptoms (eg,

Table 1 Baseline characteristics of included GPs and patients

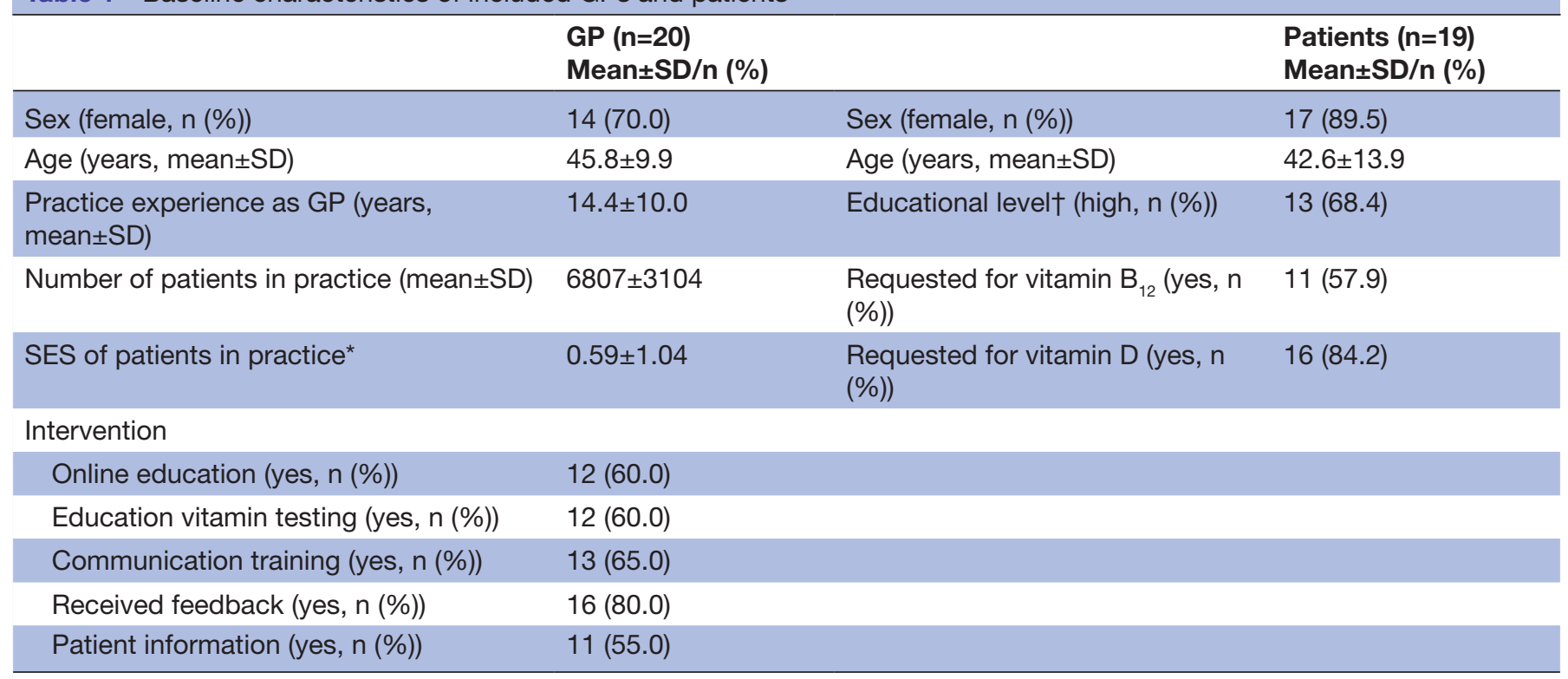

*SES data were retrieved from the SCP and linked by four digital postal codes to our data. SCP calculates social economic status scores based on information regarding education, income and position in the labour market. An SES score of 0 defines the mean socioeconomic status in the Netherlands. A score of $>0$ defines an SES higher than the mean in the Netherlands. A score of $<0$ defines an SES lower than the mean in the Netherlands.

†A high educational level was defined as an academic bachelor degree or higher.

GP, general practitioner; SCP, Social and Cultural Planning Office; SES, socioeconomic status. 
fatigue or myalgia) only in a minority of patients or if patients insisted on having their vitamin levels tested.

\section{Non-medical reasons}

Maintaining a good relationship with the patient, avoiding conflict and creating goodwill for follow-up consultations were mentioned both for vitamin $\mathrm{D}$ and $\mathrm{B}_{12}$ testing. These non-medical reasons were important arguments to order the test, if patients persisted in their request to have their vitamin $\mathrm{B}_{12}$ or $\mathrm{D}$ levels tested, despite adequate explanation by the GP.

You can't refuse every request, because that will not improve your relationship with the patient. You will create goodwill, when you agree with some requests from the patients. As a consequence, they will trust you more and they will agree with your advices in follow-up consultations, instead of refusing them. GP1, woman, 31 years, 'Creating goodwill for follow-up consultations'

\section{Influence of participation in revert study}

Most of the GPs mentioned that they reduced their vitamin $\mathrm{D}$ and $\mathrm{B}_{12}$ test ordering as a result of participation in the REVERT study. They reported investing more time during the consultation in explaining vitamin test indications and discussing reasons for not testing, after having followed the education on vitamin testing.

About half of the GPs advised their patients to supplement vitamin D instead of having their vitamin D level tested. A few GPs reported that they did not change much in their testing behaviour. They indicated that, before participation in REVERT, they rarely tested vitamin levels.

Now I tell patients that they could start with supplements if they think that there is an association between their symptoms and a vitamin deficiency. Just start with supplements.' GP11, man, 43 years, 'Advice to supplement vitamin D instead of testing'

I give patients more information and explanation at this moment. I always tested vitamin D and B12 levels in patients complaining of fatigue before I received education. I don't do that anymore.' GP7, woman, 65 years, 'Spending more time explaining'

\section{GPs' motivational factors}

Regarding the motivation to reduce unnecessary vitamin tests, three aspects could be identified: (1) ideas and attitudes towards the usefulness of reducing vitamin tests, (2) attitudes towards the effort to change testing behaviour and (3) influence of intervention on motivation to change testing behaviour.

Ideas and attitudes towards the usefulness of reducing vitamin tests

Most of the GPs considered reduction of unnecessary vitamin testing as beneficial. These GPs believed that they improved healthcare quality and cost efficiency by reducing unnecessary vitamin tests, through preventing medicalisation of patients and/or reducing healthcare costs.

\section{Attitudes towards the effort to change behaviour}

Some GPs were not motivated to change their testing behaviour because they expected the resulting reduction in healthcare costs to be disappointing. Another aspect of some GPs' negative attitude towards reducing vitamin testing was their observation that symptoms in deficient patients were resolved after they started vitamin D supplementation. One GP mentioned vitamin testing as being helpful by using a 'proven low vitamin level' as 'placebo tool', being a substrate or explanation for their symptoms.

Influence of intervention on motivation to change testing behaviour GPs mentioned that feedback of their testing behaviour in the REVERT project helped them to stay motivated to reduce unnecessary vitamin testing. For a sustainable strategy to reduce test ordering, GPs suggested retaining this feedback on testing behaviour. Individual feedback instead of feedback on the practice's performance might be more effective because it could create more insight into GPs' personal test-ordering behaviour.

I think that if you continue with over-testing vitamin levels, you are giving patients the idea that vitamin testing is very useful. When you stop over-testing vitamin levels, you will stimulate patients to reflect on their total well-being instead of only requesting laboratory testing.' GP10, woman, 48 years, 'Preventing medicalisation'

When you request laboratory tests, you have no idea about the number of requests you make. It appears to be a lot more than you think. I didn't expect that.' GP13, man, 57 years, 'Awareness of testing behaviour'

It is a kind of tool which I can use and I don't want to lose that tool. I sometime use it as placebo. I'd like to use this tool, because I think that I can help patients by saying that their symptoms might be due to a low vitamin level and that the symptoms might disappear when they start with supplements. I believe that, when using this placebo tool, I contribute to preventing patients from visiting other specialists with their vague symptoms.' GP3, man, 34 years, 'Proven low vitamin level as placebo tool'

\section{GPs' cognitive factors}

GPs mentioned cognitive barriers and facilitators for reducing the number of vitamin tests. These can be summarised in two categories: (1) influence of the REVERT intervention on GPs' knowledge and (2) conflicting medical information.

\section{Influence of intervention on GPs' knowledge}

Up-to-date knowledge about the usefulness of vitamin tests, offered through the (online) education in the REVERT study, was the most important facilitator for reducing vitamin testing according to GPs. GPs mentioned that, apart from the up-to-date knowledge, the concrete 
patient examples and the background information about guideline-based indications for vitamin testing and treatment discussed in the (online) education in the REVERT study also contributed to changing testing behaviour regarding vitamin $\mathrm{D}$ and $\mathrm{B}_{12}$, because it was helpful in giving explanations to patients about the usefulness of vitamin testing.

Lack of repetition of the information was mentioned as the most important cognitive barrier to remembering, with the risk of falling back into old patterns of test ordering. Four GPs mentioned that it was difficult to remember all the information received during the single moment of (online) education. Nine GPs mentioned that it was easier to remember all the information if they had received other education about this subject in the past or frequently discussed the topic in meetings with colleagues.

\section{Conflicting medical information}

Conflicting results and recommendations from other information sources were mentioned as the most important barrier to reducing the number of vitamin tests requested by GPs. About half of the GPs mentioned these conflicting results in the literature about the association between symptoms and vitamin levels as a problem in building up their argumentation during the patient consultation. They also mentioned that global recommendations sometimes differ from national guideline recommendations. These intercountry differences were mentioned as a reason for discussion with patients. Some GPs therefore thought it difficult to resist vitamin test requests from patients, especially when patients' 'knowledge' seemed to be better than their own knowledge on this topic.

It is still very difficult to translate the information that you received from (online) education to an explanation for a very demanding patient in $10 \mathrm{~min}$. Especially when the patient has searched for a lot of different articles that emphasise the importance of vitamin testing.' GP5, woman, 37 years, 'GP does not feel confident enough about knowledge'

As part of a sustainable strategy to reduce vitamin testing in general practice, GPs mentioned the need for an overview of up-to-date knowledge about vitamin testing in a national guideline or protocol. GPs thought that such a protocol would make it easier for health professionals to quickly search for answers when unable to recall the information from previous (online) education.

So, I needed some kind of protocol that included the 10 most important things that I had learnt during the online education. I noticed that I had difficulty recalling information from previous sessions and therefore returned.' GP4, woman, 38 years, 'Need for a protocol'

\section{Social factors affecting GPs' testing behaviour}

GPs reported the following social factors affecting their testing behaviour: (1) interaction with patients, (2) attitudes of other health professionals, and (3) influence of media and society.

\section{Interaction with patients}

GPs indicated that good communication skills are needed to provide explanations and to convince patients that vitamin tests are not always necessary. GPs also mentioned that a low education level and language barriers made it more difficult to communicate and that they regarded these as barriers to providing a good explanation to patients on the limited usefulness of vitamin testing.

GPs mentioned that is was easier to convince patients with whom they had a long relationship compared with patients who were relatively new in their general practice. One GP mentioned using her seniority, due to her age, making it easier to convince patients to agree with non-testing.

\section{Attitudes of other health professionals}

Six GPs mentioned that their partner GPs in the practice were less motivated to reduce unnecessary vitamin tests or had different opinions about vitamin testing than themselves. Also, the presence of locum doctors in the practice was mentioned as a barrier to reducing the number of vitamin tests requested, because locums were found to request vitamin tests more often. In some practices, assistants were able to request vitamin levels on their own initiative, limiting the reduction in vitamin testing. On the other hand, four GPs reported that all the GPs in their practice had the same thoughts and restrictive methods regarding vitamin testing. Furthermore, it was considered helpful if other health professionals, for example, GPs' assistants, had up-to-date knowledge about vitamin testing through education in order to provide patients with the same message on the limited usefulness of vitamin testing.

It is important that the assistants have the same knowledge as the GPs, because they are asked the most questions about vitamin testing.' GP19, man, 35 years, 'Up-to-date knowledge among GPs' assistants

\section{Influence of media and society}

Another reported factor that made it difficult to reduce vitamin tests is the information spread about the suggested importance of unrestricted vitamin $\mathrm{D}$ and $\mathrm{B}_{12}$ tests by other healthcare professionals, the social media or other patients. In line with this, GPs suggested that more support from colleagues, media and society should be part of a sustainable strategy to reduce unnecessary vitamin tests. GPs specifically mentioned the need for reliable information resources for patients. 


\section{Patients' motivational factors}

Two components of patients' motivation to change behaviour could be distinguished: (1) attitudes towards GPs and (2) attitudes towards vitamin testing.

\section{Attitudes towards GPS}

About half of the patients mentioned that they had a negative attitude on this subject towards their GP. These patients were convinced that their GP did not have enough knowledge about vitamins (tests); this resulted in distrust and dissatisfaction with the information provided and the decisions made by their GP regarding vitamin testing.

I decided to look up all the information I wanted to know, because my GP couldn't tell me much about it, that was a pity. I think that I do know more about vitamin testing than my GP knows.' P12, woman, 40 years, 'GP does not have enough knowledge'

\section{Attitudes towards vitamin testing}

Most of the patients also had a negative attitude towards a policy of 'not testing' and even suggested that it would be better if GPs increased vitamin testing and paid more attention to vitamin deficiencies. In line with a negative attitude towards not testing, about $50 \%$ of the patients reported not seeing any alternative for vitamin blood tests. Moreover, they stated their dissatisfaction with GPs who were unwilling to test their vitamin levels. Two patients mentioned that they would keep asking their GP for vitamin tests until their request was met.

The GP always disagrees with my requests for vitamin testing, saying: 'I don't think that vitamin deficiency is the problem'. I have to be very demanding and in the end I get what I want.' P5, woman, 53 years, 'Keep asking the GP for vitamin testing'

Some patients mentioned that they would accept a satisfactory explanation from their GP about the reasons for not testing if the GP disagreed with their vitamin test request. Two patients suggested that health professionals with a background in complementary medicine could be consulted as an alternative for having vitamin levels in their blood tested when the GP disagreed with their request.

\section{Patients' cognitive factors}

Two components of cognition and knowledge about vitamin (testing) can be identified in patients: (1) thoughts and attitudes regarding information sources and (2) patients' reasons for wanting to be tested.

\section{Thoughts and attitudes regarding information sources}

Most of the patients used the internet to search for information about vitamins. Five patients had read information about vitamins in books and magazines. Psychological symptoms, myalgia and fatigue were the most frequently mentioned symptoms associated with vitamin $\mathrm{D}$ and $\mathrm{B}_{12}$ deficiencies. Patients mentioned that the information that they found on the association between vitamin deficiencies and symptoms gave them an explanation for their symptoms.

'I decided to look online for more information and I recognised a lot of my symptoms in the stories that I read on the internet.' P17, woman, 31 years, 'Online information sources'

Patients thought it confusing that there are differences between reference levels and advices between countries and study results. They mentioned that these differences made it more difficult to believe that their GP's reference levels were correct.

\section{Patients' reasons for wanting to be tested}

Patients' main reason for asking their GP to have their vitamin levels tested was fatigue. Other reasons mentioned were depressive symptoms, weight loss and myalgia. A vegetarian or vegan diet was also mentioned as a reason for having a vitamin $\mathrm{B}_{12}$ test. Some patients mentioned that a history of vitamin deficiency strengthened their request to have their vitamin $\mathrm{D}$ and/or $\mathrm{B}_{12}$ levels tested.

\section{DISCUSSION}

\section{Summary of key findings}

In this qualitative analysis, we found a wide spectrum of patient-related and GP-related perceptions and attitudes that affect vitamin test ordering in clinical practice (summarised in figure 1). The most important factors hampering vitamin test reduction programmes are the mismatch between patients and medical professionals regarding the presumed indications for testing for vitamin $\mathrm{D}$ and $\mathrm{B}_{12}$, differences in motivation, and the GPs' tendency to avoid conflict. The most important facilitator for vitamin test reduction programmes is updating GPs' knowledge about test indications, in combination with improving their awareness of their individual test behaviour.

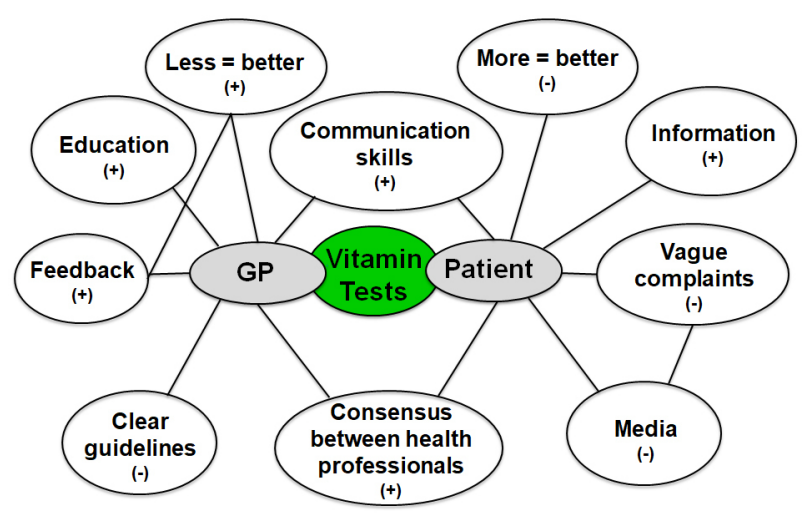

Figure 1 Patient-related and GP-related perceptions and attitudes affecting vitamin test ordering in clinical practice. GP, general practitioner. 


\section{Reasons for testing differed between patients and GPs}

For patients, the most important reasons to ask for vitamin testing were (non-specific) medical symptoms based on information found on the internet and confirmed by other media, contacts and sometimes other healthcare professionals. GPs, however, mentioned being aware of the lack of indication for vitamin testing when patients presented with non-specific medical symptoms.

GPs used information from the (online) project education to rebut patients' ideas and explain about the limited usefulness of vitamin testing. Conflicting results and recommendations between different information sources result in confusion about indications and the usefulness of vitamin testing among both GPs and patients, creating discussion between GP and patients. A difference between patients and GPs in their motivation to change testing behaviour was also identified. Whereas most GPs were very motivated to reduce vitamin testing, most patients suggested that it would be better if GPs tested more frequently for vitamin deficiencies in general practice.

Another barrier to reducing the number of vitamin tests was GPs' tendency to avoid conflict and to satisfy patients in order to foster good relationships with patients. In line with this, good communication skills facilitated GPs in discussing and explaining the limited usefulness of vitamin testing to patients.

Other facilitators for reducing the number of vitamin tests according to GPs were consensus between healthcare professionals and ongoing feedback on testing behaviour, but almost all GPs mentioned up-to-date knowledge about the usefulness of vitamin testing through education as the most important facilitator for reducing vitamin tests.

Following from this, to enable GPs to recall information, a reliable overview of the evidence and recommendations regarding vitamin testing is warranted. GPs mentioned that this knowledge should also be available to other healthcare professionals and patients in order to create unanimity about the usefulness of vitamin tests. GPs also suggested getting regular individual feedback about their testing behaviour to keep them motivated to test only when necessary and to have a tool to remind them to change their testing behaviour.

\section{Results in context}

Patients and GPs having conflicting information was one of the main barriers to reducing unnecessary vitamin testing. In line with our results, previous research has highlighted that not only health professionals but also the media are key information providers on this topic for patients. ${ }^{20}$ A media content analysis showed that news articles linked vitamin $\mathrm{D}$ to a wide range of health conditions without conclusive scientific evidence. ${ }^{21}$ As reflected by our study as well as previous research, this has resulted in confusion regarding the usefulness of vitamin testing, among both patients and GPs. ${ }^{92}$ Moreover, GPs' information sources also present conflicting results, reinforcing this confusion. To counter this, previous research highlighted the need for clear information that reflects the actual state of knowledge and for ongoing research for both healthcare professionals and patients. ${ }^{9} 1020$ Similarly, GPs in this study mentioned that clear guidelines for patients and GPs regarding vitamin testing would help them in discussions with their patients. In line with this, in this study education was found as one the most important facilitators for reducing vitamin testing. Previous research showed that education and communication through electronic educational codified comments might improve vitamin requests. ${ }^{23}$ In addition, strategies for reducing unnecessary vitamin testing require continuous education, because the intervention effect of education seems to decrease over time. ${ }^{24}$

Feedback on testing behaviour was found to be another important facilitator for reducing the number of unnecessary vitamin tests. This is in line with an RCT that showed that feedback of requesting rates was an effective strategy for reducing laboratory testing in primary care..$^{25}$ The results of a systematic review suggest that feedback may be more effective when it is provided more than once and when it includes both measurable targets and an action plan. ${ }^{26}$ These suggestions could be useful for implementing feedback on testing behaviour in the future. GPs suggested that feedback on individual GP behaviour might be more effective than feedback on practice level. Such individual feedback might contribute to the measurability of targets and a personalised action plan.

\section{Strengths and limitations}

This is the first study to use semistructured interviews to explore the barriers and facilitators for reducing the number of (unnecessary) vitamin $\mathrm{D}$ and $\mathrm{B}_{12}$ laboratory tests ordered. The qualitative approach and the use of open coding based on a broad theoretical framework allowed us to highlight all the different aspects behind the complexity of reducing vitamin testing. The validity and reliability of this study were strengthened by including patients from a broad range of backgrounds, as well as GPs from 20 different practices.

Still, a few limitations need to be addressed. First, participating GPs were affiliated to a research network and therefore might not have been representative of all GPs in the Netherlands. Next, patients were invited for the interviews by their GPs; this creates a potential bias arising from the selection of, for example, more outspoken patients. However, patient characteristics (table 1) show large variation in age, sex and educational level, making inclusion of different patient perspectives likely. Finally, even though the same interview guide was used, the interviews in this study were performed by two different researchers, who may have had differences in their interviewing style that may have influenced participants' responses.

\section{Recommendations}

From a GP's perspective, a sustainable reduction in vitamin test requests in primary care requires the following steps: (1) updating GPs' knowledge through (online) 
education, (2) guidelines with clear and uniform recommendations on prevailing indications for vitamin testing and supplementation for all healthcare professionals, and (3) regular (individual) feedback on GPs' test behaviour.

From a societal perspective, access to clear and reliable information on vitamin testing for the population is needed, from trustful sources. In addition, the spread of non-evidence-based information through lay media should be challenged. Further research is required to measure the effect of these strategies on reducing vitamin testing.

\section{CONCLUSION}

In conclusion, conflicting information about the usefulness of vitamin testing, differences in motivation between patients and GPs, as well as GPs' tendency to avoid conflict and to satisfy patients are important barriers to reducing the number of vitamin tests. Nevertheless, updating GPs' knowledge, feedback on GPs' testing behaviour and guidelines with clear recommendations for all healthcare professionals (including patient information) on prevailing indications for vitamin testing and supplementation could facilitate a sustainable reduction in vitamin testing in primary care.

Acknowledgements We thank the entire Reducing Vitamin Testing in Primary Care Practice team for their diligence, expertise and enthusiasm. Finally, we are indebted to all patients and general practitioners who consented to be interviewed, without whom this study would not have been possible.

Contributors $\mathrm{HH}, \mathrm{HvdB}, \mathrm{BM}, \mathrm{AB}, \mathrm{EdS}, \mathrm{PB}, \mathrm{NdW}$, and SvV conceived the study idea and designed the study. HH, HvdB, BM, AB, EdS, PB, NdW, and SvV helped to develop the protocol and coordinated the collection of all data. $\mathrm{HH}$ and $\mathrm{HvdB}$ interpreted the data and performed the analyses, with help from BM, AB, EdS, $\mathrm{PB}, \mathrm{NdW}$, and SvV. HH wrote the first draft of the manuscript, and all mentioned coauthors critically revised the manuscript. All authors had full access to all of the data (including statistical reports and tables) in the study and can take responsibility for the integrity of the data and the accuracy of the data analysis.

Funding The study was funded by the Citrien Fund, a national programme of the Dutch government initiated in 2015 (ie, 'Do or don't' programme) to reduce lowervalue services (grant number 8392010023). The funding source was not involved in the design, conduct, analysis and interpretation of the data, nor in the writing and the decision to submit the paper.

Competing interests None declared.

Patient consent for publication Not required.

Provenance and peer review Not commissioned; externally peer reviewed.

Data availability statement Data are available upon reasonable request.

Open access This is an open access article distributed in accordance with the Creative Commons Attribution Non Commercial (CC BY-NC 4.0) license, which permits others to distribute, remix, adapt, build upon this work non-commercially, and license their derivative works on different terms, provided the original work is properly cited, appropriate credit is given, any changes made indicated, and the use is non-commercial. See: http://creativecommons.org/licenses/by-nc/4.0/.

ORCID iD

H. Hofstede http://orcid.org/0000-0002-9487-6841
REFERENCES

1. Sattar $\mathrm{N}$, Welsh $\mathrm{P}$, Panarelli $\mathrm{M}$, et al. Increasing requests for vitamin $\mathrm{D}$ measurement: costly, confusing, and without credibility. The Lancet 2012;379:95-6.

2. Franken P, Geutjes P, van den Ouweland J, et al. Diagnose van vitamine-B12-tekort. Huisarts Wet 2015;58:530-1.

3. Medicare payments for clinical diagnostic laboratory tests in 2016 : year 3 of baseline data, 2017. Available: https://oig.hhs.gov/oei/ reports/oei-09-17-00140.asp

4. Bilinski K, Boyages S. Evidence of overtesting for vitamin D in Australia: an analysis of 4.5 years of Medicare benefits schedule (Mbs) data. BMJ Open 2013;3:e002955.

5. LeFevre ML, LeFevre NM. Vitamin D screening and supplementation in community-dwelling adults: common questions and answers. Am Fam Physician 2018;97:254-60.

6. Bindels PJE. [Vitamin D: what to do with it?]. Ned Tijdschr Geneeskd 2015;159:A8837.

7. Langan RC, Goodbred AJ. Vitamin B12 deficiency: recognition and management. Am Fam Physician 2017;96:384-9.

8. Moynihan R, Doust J, Henry D. Preventing overdiagnosis: how to stop harming the healthy. BMJ 2012;344.

9. Kotta S, Gadhvi D, Jakeways N, et al. "Test me and treat me"-attitudes to vitamin $D$ deficiency and supplementation: a qualitative study. BMJ Open 2015;5:e007401.

10. Gowda U, Smith BJ, Wluka AE, et al. Vitamin D testing patterns among general practitioners in a major Victorian primary health care service. Aust N Z J Public Health 2016;40:144-7.

11. Rodriguez-Borja E, Corchon-Peyrallo A, Barba-Serrano E, et al. "Send \& hold" clinical decision support rules improvement to reduce unnecessary testing of vitamins A, E, K, B1, B2, B3, B6 and C. Clin Chem Lab Med 2018;56:1063-70.

12. Michiels-Corsten M, Donner-Banzhoff N. Beyond accuracy: hidden motives in diagnostic testing. Fam Pract 2018;35:222-7.

13. Hajjaj FM, Salek MS, Basra MKA, et al. Non-Clinical influences on clinical decision-making: a major challenge to evidence-based practice. J R Soc Med 2010;103:178-87.

14. O'Brien BC, Harris IB, Beckman TJ, et al. Standards for reporting qualitative research: a synthesis of recommendations. Acad Med 2014;89:1245-51.

15. Charmaz K. Constructing grounded theory: a practical guide through qualitative analysis. Sage, 2006.

16. Grol R, Wensing M, Bosch M, et al. Theories on implementation of change in healthcare. In: Improving Patient Care: The Implementation of Change in Health Care, 2013: 18-39.

17. Social and Cultural office [Sociaal en Cultureel Planbureau], Social economic factors [Statusscores], 2009.

18. Guest G, Bunce A, Johnson L. How many interviews are enough? an experiment with data saturation and variability. Field Methods 2006;18:59-82.

19. Zamawe FC. The implication of using NVivo software in qualitative data analysis: evidence-based reflections. Malawi Med J 2015;27:13-15

20. Deschasaux M, Souberbielle J-C, Partula V, et al. What do people know and believe about vitamin D? Nutrients 2016;8:718.

21. Caulfield T, Clark MI, McCormack JP, et al. Representations of the health value of vitamin D supplementation in newspapers: media content analysis. BMJ Open 2014;4:e006395.

22. Bennett K, Frisby BN, Young LE, et al. Vitamin D: an examination of physician and patient management of health and uncertainty. Qual Health Res 2014;24:375-86.

23. Salinas M, López-Garrigós M, Flores E, et al. Education and communication is the key for the successful management of vitamin D test requesting. Biochem Med 2015;25:237-41.

24. Miyakis S, Karamanof G, Liontos M, et al. Factors contributing to inappropriate ordering of tests in an academic medical department and the effect of an educational feedback strategy. Postgrad Med $J$ 2006;82:823-9.

25. Thomas RE, Croal BL, Ramsay C, et al. Effect of enhanced feedback and brief educational reminder messages on laboratory test requesting in primary care: a cluster randomised trial. The Lancet 2006;367:1990-6.

26. Ivers N, Jamtvedt G, Flottorp S, et al. Audit and feedback: effects on professional practice and healthcare outcomes. Cochrane Database Syst Rev 2012;154. 